\title{
Prolégomènes à une études historique des rapports entre l'État et le droit dans la société québécoise, de la fin du XVIII ${ }^{\mathrm{e}}$ siècle à la crise de 1929
An Introduction to the Historical Study of the Relationship between the State and the Law in Quebec Society, from the End of the 18th Century to the Crisis of 1929

\section{Jean-Marie FECTEAU}

\section{Volume 18, numéro 1, avril 1986}

Droit et pouvoir, pouvoirs du droit

URI : https://id.erudit.org/iderudit/001241ar

DOI : https://doi.org/10.7202/001241ar

Aller au sommaire du numéro

\section{Éditeur(s)}

Les Presses de l'Université de Montréal

ISSN

0038-030X (imprimé)

1492-1375 (numérique)

Découvrir la revue

Citer cet article

FECTEAU, J.-M. (1986). Prolégomènes à une études historique des rapports entre l'État et le droit dans la société québécoise, de la fin du XVIII ${ }^{\mathrm{e}}$ siècle à la crise de 1929. Sociologie et sociétés, 18(1), 129-138.

https://doi.org/10.7202/001241ar

\section{Résumé de l'article}

La prépondérance actuelle de la régulation sociale sous une forme juridique présuppose l'existence de cet appareil centralisé qu'est l'État. Le modèle libéral ne sépare pas réellement le public et le privé: libéralisation du marché et réglementation étatique y vont de pair. Dans cette optique, l'auteur examine le développement historique concomitant du droit et de l'État modernes au Québec. Déclin du modèle colonial d'abord (1760-1830), quand la crise du système normatif devient très vite une question de souveraineté: la faculté royale de produire et d'appliquer le droit se heurte à la notion révolutionnaire de la légitimité démocratique. Ensuite (1830-1885), autonomisation relative du droit comme mode de normalisation et légitimité démocratique de l'État et de ses appareils de réglementation et de gestion. Enfin (1885-1930), remise en question par le mouvement ouvrier de l'idéologie de la séparation du public et du privé et développement rapide du droit public, au point de remettre bientôt en cause le droit privé comme catégorie juridique autonome.
Tous droits réservés @ Les Presses de l'Université de Montréal, 1986
Ce document est protégé par la loi sur le droit d'auteur. L'utilisation des services d'Érudit (y compris la reproduction) est assujettie à sa politique d'utilisation que vous pouvez consulter en ligne.

https://apropos.erudit.org/fr/usagers/politique-dutilisation/ 


\section{Prolégomènes à une étude historique des rapports entre l'État et le droit dans la société québécoise, dle la fin du XVIII ${ }^{\mathrm{e}}$ siècle à la crise de 1929}

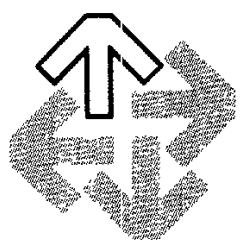

JEAN-MARIE FECTEAU

Au cours des dernières années, on a assisté, au Canada, à un remarquable développement des recherches en matière d'histoire du droit ${ }^{1}$. On ne peut cependant en dire autant de l'historiographie touchan1. le développement de l'appareil d'État. Mises à part les études d'histoire administrative ${ }^{2}$, on sait relativement peu de choses sur les conditions de développement de l'État québécois et sur les modalités de son insertion dans les rapports sociaux depuis la fin du XVIII ${ }^{\text {e }}$ iècle $^{3}$. Il est d'ailleurs remarquable de noter la séparation quasi absolue entre le champ de l'histoire du droit et les études qui s'attachent à l'évolution de l'État. C'est pourtant dans la mise en rapport systématique de ces deux types de questionnements que réside, selon nous, l'espoir d'un dépassement des perspectives trop étroites où ils restent confinés. Ce court essai doit être vu avant tout comme une première tentative d'en arriver à une réflexion d'ensemble sur les rapports entre État et droit dans l'histoire québécoise.

\section{L'ÉTUDE HISTORIQUE DU PHÉNOMÈNE JURIDIQUE}

La réflexion sur le droit et son histoire subit, notamment dans les sociétés occidentales, un ensemble de contraintes spécifiques à ce champ. La première contrainte tient au problème de la «définition» du droit. Les tentatives de définir le droit comme contenu, c'est-à-dire comme ensemble de normes qui structurent une formation sociale donnée, se heurtent au problème du caractère extrêmement volatile de toute norme. Plus précisément, si une «norme» prescrit ou prohibe un comportement spécifique dans une circonstance donnée, sa durée, son emprise, son caractère

1. Le recueil publie en deux volumes par D.H. Flaherty, Essays in the History of Canadian Law (Toronto, University of Toronto Press, 1981 et 1983), est un bon indice de ce phénomène.

2. Les travaux de J.E. Hodgetts et de J.I. Gow demeurent encore les meilleures références en ce domaine. Voir notammerit J.E. Hodgetts, Pioneer Public Service. Toronto, University of Toronto Press, 1955; J.I. Gow, «L'histoire de l'administration publique québécoise», Recherches sociographiques, vol. 16, septembre-décembre 1975, pp. 385-411; R. Bolduc et J.I. Gow, «Environment and Administration: Quebec, 1867-1980», O.P. Dwivedi (édit.), The Administrative State in Canada, Toronto, University of Toronto Press, 1981, pp. 31-62.

3. À titre d'exemple. il n'existe pas, à notre connaissance, une seule étude sur le rôle du Conseil exécutif au BasCanada de 1791 à 1840 . Or il s'agit du principal instrument de gestion publique dans la colonie à l'époque. La série RG4Al, aux Archives publiques du Canada (Correspondance du secrétaire civil, 1760-1841), n'est pas encore inventoriée. Elle a d'ailleurs été fort peu utilisée jusqu'ici. 
contraignant, le type de sanction qui y est appliquée sont d'une telle diversité qu'on peut difficilement les subsumer sous un concept unitaire, fût-il aussi large que celui de «droit». Il devient donc nécessaire de faire intervenir des caractères discriminants qui puissent exclure de la sphère juridique une vaste partie de ces normes sociales opératoires. L'élaboration de ces critères (caractère contraignant, sanction collective ou publique, appareil distinct de mise en application, reconnaissance générale, adhésion majoritaire, monopole étatique, etc.) amène certes à isoler un champ spécifique de régulation, mais c'est au prix d'une séparation dont la justification théorique est trop souvent laissée dans l'implicite. Une grande part de l'histoire du droit repose sur ce non-dit d'un champ qui se veut autonome ${ }^{4}$, et qui ne trouve sa légitimité véritable que dans la notion moderne d'un droit autonomisé et relativement bien circonscrit.

D'autre part, on tend de plus en plus, dans la lignée de E. Pashukanis, à saisir le droit comme forme structurelle ${ }^{5}$ qualifiant une formation sociale donnée. O. Correas, notamment, décrit le droit comme «une des formes d'existence des relations sociales ${ }^{6} »$. Cette approche a l'immense avantage de poser, immédiatement et clairement, au-delà du contenu de la norme, le problème de l'insertion de la forme juridique dans la régulation globale d'une société, et d'ébranler du même coup la seconde contrainte qui pèse sur toute recherche touchant le droit: la contrainte disciplinaire.

En effet, si l'histoire du droit a été longtemps la chasse gardée des juristes, c'est bien sûr que les technicalités de l'analyse juridique et la spécificité des sources rebutaient - et rebutent encore - les historiens(nes). Mais il faut aussi mettre en cause la cristallisation du droit dans un appareil spécialisé de production de norme et de sanction, de même que la relative monopolisation professionnelle qui s'ensuit: le droit prend ainsi l'apparence trompeuse d'un domaine spécifique pouvant être distingué de la réalité sociale qui $l^{\prime}$ «entoure?», domaine où ne peuvent se mouvoir à l'aise que ces spécialistes que sont les juristes".

Or, la réflexion sur le droit comme forme d'existence de la sociabilité, et non comme ensemble de règles spécifiques, pose des défis nouveaux à la recherche historique. À l'étude, dominante jusqu'ici, de la logique de développement des règles juridiques", tend à se greffer de plus en plus une réflexion intégrée sur le rôle historique de la forme juridique dans les mutations sociétales ${ }^{10}$. C'est ainsi qu'après une trop longue éclipse, les recherches et analyses diachroniques de Marx, de Durkheim, de Weber apparaissent de nouveau dans toute leur richesse... et leurs limites.

Cette réflexion d'ordre diachronique débouche sur un constat troublant: la forme d'existence normative que constitue le droit apparaît comme une forme historique spécifique. Ce qui implique que l'analyse de la succession des modes de régulation ne peut se satisfaire d'un concept atemporel de droit comme ensemble de normes ou, même, comme forme sociale de réglementation. Autrement dit, l'histoire, comme mouvement temporel, est réfractaire à la recherche, d'ordre téléologique, d'une entité abstraite qui se donnerait les formes éternelles du droit. Replacé dans sa perspective historique globale, le droit n'apparaît plus que comme une des formes que peut prendre la régulation des comportements sociaux. Cette forme résulte de la cristallisation, historiquement déterminée, d'un ensemble de rapports sociaux spécifiques. De fait, nous posons comme hypothèse centrale que la prépondérance actuelle de normes de régulation sous une forme juridique présuppose le développement d'un appareil centralisé de production, d'interprétation, d'application et de sanction de norme. Cet appareil est l'État.

4. Les travaux de M. Weber et de E. Pashukanis sont, en ce sens, de passionnantes exceptions.

5. Voir O. Correas, «Le droit comme «forme» sociale», Procès, n" 10, 1982, pp. 13-56; 1.D. Balbus «Commodity Form and Legal Form: An Essay in the 〈Relative Autonomy of the Law〉», C.R. Reasons et R.M. Rich (édit.), The Sociology of Law. A Conflict Perspective. Toronto, Butterworths, 1978, pp. 73-90

6. O. Correas, op. cit.. p. 18

7. À tel point que le marxisme structuraliste, à la suite d'Althusser, l'a assigné au niveau de la «superstructure» sociale.

8. La médecine a connu un sort semblable

9. On trouvera des exemples classiques de cette démarche dans S.F.C. Milsom, Historical Foundations of the Common Law, London, Butterworths, 1981 et J.H. Baker, An Introduction to English Legal History, London. Butterworths, 1979 .

10. C'est. selon moi. à cette tendance profonde qu'il faut attribuer l'énorme impact du livre de M.J. Horwitz, The Transformation of American Law. Cambridge, Harvard Unversity Press, 1977. Voir cependant les réticences de D.G. Bell, «The Birth of Canadian Legal History». University of New Brunswick Law Journat. vol. 33, 1984, pp. 312-318. 


\section{LA PLACE DE L'ÉTAT ET LE DROIT}

Au cours des dix dernières années, on a assisté à un remarquable développement des analyses et travaux consacrés au rôle de l'État dans les sociétés contemporaines ${ }^{11}$. Cependant, trop peu de ces études s'interrogent sur la place du droit dans l'expansion des activités étatiques, si ce n'est de façon incidente ${ }^{12}$. Le phénomène est encore plus notable si on se tourne du côté des études historiques récentes en cette matière ${ }^{13}$. A l'inverse, la réflexion de type juridique n'interroge trop souvent le rôle de l'État qu'au niveau de la philosophie ${ }^{14}$ ou sous l'angle étroit du droit public ${ }^{15}$.

Tout se passe en fait comme si État et Droit ne pouvaient avoir de rapports que comme implicites l'un de l'autre: le Droit ne peut se penser sans un pouvoir d'État plus ou moins mal défini, et l'État ne peut remplir sa fonction propre que dans les formes de la légalité. Ce que fait l'État est de droit, alors que ce qui donne sens au droit est l'État. C'est dans cette dialectique complexe que se matérialise la régulation des sociétés modernes. Dans la mesure où l'État règlemente de plus en plus les rapports sociaux, la légalité prend les formes complexes et multiples qu'on lui connait aujourd'hui.

Plus précisément, le droit se présente comme le mode d'expression spécifique du pouvoir d'État: la norme juridique a l'État comme frontière et comme possible. La régulation sociale prend une forme juridique lorsqu'elle est médiatisée par l'instance publique ${ }^{16}$. L'histoire du droit, audelà du contenu de la norme et de ses modalités d'existence, est donc aussi l'histoire de l'État, de la constitution d'une instance centrale de régulation qui en vient à assurer son emprise, directe ou indirecte, sur tous les aspects essentiels de la vie sociale. Pour que cette emprise soit réelle et non pas seulement symbolique, il faut que se développe un appareil, un ensemble de moyens matériels d'action qui assure non seulement la présence, mais l'efficace particulière du pouvoir public. En ce sens, l'histoire du développement de l'État implique à la fois la «juridicisation» des rapports sociaux et la mise en place d'un appareil public de régulation. Les grandes catégories du droit moderne ne sont en fait, dans cette optique particulière, que des modalités d'insertion de la règle publique dans les rapports sociaux. Par exemple le droit civil ou "privé», comme catégorie juridique moderne, implique certes que les rapports de propriété et entre individus (famille, possession et échange de biens, etc.) obéissent à des règles pré-établies (codifiées ou non) de nature privée, mais ces mêmes règles ne peuvent se penser sans la sanction publique préalable qui leur donne, à une certaine étape du développement historique, un caractère contraignant ${ }^{17}$. La généalogie de ce droit «privé» illustre bien la lente récupération par le pouvoir royal des coutumes locales, et leur insertion plus ou moins brutale, aux XVIII et XIX ${ }^{\circ}$ siècles, dans un cadre public repensé, sous les formes de la codification ou de la consolidation ${ }^{18}$.

En d'autres termes, on assiste, à partir de la fin $\mathrm{du} X \mathrm{XVII}{ }^{\mathrm{C}}$ siècle surtout, à une mutation brutale des critères d'opération du droit et de l'État. Cette mutation est permise par un bouleversement radical des fondements de la légitimité politique. Les révolutions populaires, les soulèvements de type nationalitaire, l'organisation de plus en plus massive des classes ouvrières imposent un réa-

11. La bibliographie est énorme. On en trouvera un bilan fort valable chez B. Jessop, The Capitalist State, N.Y., New York. University Press, 1982

12. Voir par exemple N. Poulantzas, l'État, le Pouvoir, le Socialisme, Paris, Presses universitaires de France, 1978, pp. 83-101; D. Charvet, "Crisc de la Justice, crise de la loi, crise de l'État'?», N. Poulantzas (dir), la Crise de l'État, Paris, Presses universitares de France, 1976. pp. 261-292.

13. Notamment la remarquable étude de R. Delorme et C. André, l'État et l'économie, Paris, Seuil, 1983. Voir aussi L. Fontvieille, «Évolution et croissance de l'État français, 1815-1969", Économies et sociétés, vol. 10, n" 9-12, 1976, pp. 1654-2144.

16. A. Brimo, les Grands Courants de la philosophie du droit et de l'État, Paris, Pedone, 1967.

15. On en trouve un exemple québécois chez. L. Baudoin. les Aspects généraux du droit public de la province de Québec, Paris, Dalloz, 1965.

16. C'est pourquoi il est si difficile de situer le «droit» dans une société où la décentralisation et l'éclatement des pouvoirs réduit à une quasi-fiction la souveraineté du pouvoir public. C'est le cas notamment de la société médiévale. Voir A.J. Gourevitch, les Catégories de la culture médiévale, Paris, Gallimard, 1983, pp. 155-212.

17. Le fait que l'origine de ces règles est souvent à retrouver dans les compromis locaux, en marge du pouvoir d'État (c'est le cas des coutumes) ne change rien au fait que leur mise en ouvre est assurée, à partir d'un certan stade, par l'État.

18. La situation spécifique des pays de «Common Law» donne à ce mouvement général une teinte particulière, à cause notamment de la résistance du pouvoir judiciaire. Voir A. Harding, A Social History of English Law, London, Penguin, 1966, pp. 330-388; A.H. Manchester. A Modern Legal History of England and Wales, 1750-1950, London, Butterworths, 1980, pp. 10-49; G.R. Rubin et D. Sugarman (édit.), Law, Economy and Society. Essays in the History of English Law, Abington, Professional Books, 1984. 
ménagement majeur de l'ordre politique et de la logique d'opération du droit. La démocratie bourgeoise n'est que cet agencement spécifique où un «espace public» ${ }^{19}$, fondé sur la volonté dite populaire, permet l'autonomisation d'une sphère privée dominée par la concurrence et le libre marché. On a trop longtemps négligé le fait que cet agencement nouveau est essentiellement politique, qu'il ne postule aucunement une séparation réelle entre le public et le privé, mais qu'il s'agit bien au contraire d'une économie politique de la régulation où la légitimité du pouvoir collectif assumé par l'État est centrale, où le marché et la libre concurrence sont, en fait, réglementés, quadrillés, balisés, arbitrés et reproduits par la puissance publique matérialisée dans l'État libéral ${ }^{20}$. La concomitance entre l'expansion du libre marché et celle de l'appareil administratif ${ }^{\text {"I }}$ n'a rien, en ce sens, d'une cö̈ncidence: il s'agit de deux manifestations complémentaires d'un même phénomène: «libéralisation» du marché et des échanges et réglementation étatique forment les contours d'une régulation sociale spécifique, où la personne assume les facettes contradictoires mais cumulatives du producteur et du citoyen, de l'individu et de l'être social, de l'adversaire et du collectif. Cette dialectique est historiquement déterminée par la constitution d'un espace public de la représentation et d'un espace privé de l'échange, espaces dont la fusion, au XVIII ${ }^{e}$ et surtout au XIX ${ }^{e}$ siècle, a donné naissance aux sociétés modernes, espaces dont la forme structurelle majeure est le droit, le cadre juridique qui se déploie selon les catégories réglementaires qui nous sont maintenant familières: privé/public, civil/criminel, objectif/subjectif, choses/personnes ${ }^{22}$. Dans tout l'Occident, le demisiècle qui s'étend de 1830 à 1880 environ est déterminant dans la structuration du modèle libéral de régulation. Les capacités d'intervention étatique prennent à cette époque leur forme administrative moderne alors que, simultanément, le développement du positivisme et du formalisme juridiques donne au droit son caractère autonome contemporain.

La mise au point théorique qui vient d'être esquissée nous permet maintenant d'amorcer l'étude historique du développement concomitant du droit et de l'État modernes à partir de la fin du XVIII siècle. Pour ce faire, nous prendrons comme terrain empirique le cas du Québec, en nous attachant à voir quelles sont les circonstances spécifiques qui président à ce développement en situation coloniale et dans le contexte fédéral.

\section{LE CAS DE LA SOCIÉTÉ QUÉBÉCOISE: QUELQUES JALONS HISTORIQUES}

Les conditions de développement de l'État au Québec et les mutations de la forme juridique dans laquelle il inscrit son action sont une des trop nombreuses «terra incognita» de notre historiographie. Il n'est donc question ici que de poser quelques jalons ou repères pour une analyse historique qui reste, dans l'ensemble, à entreprendre. Du modèle politique et juridique colonial à la grande crise de 1929 , on assiste à un bouleversement radical du cadre normatif dans lequel se meut la société québécoise. On peut distinguer trois étapes majeures dans cette évolution.

\section{A. LE DÉCLIN DU MODÈLE COLONIAL (1760-1830)}

Après la conquête et l'acquisition par l'Angleterre de la Nouvelle-France, un régime politique et juridique assez singulier est mis en place. Les conditions de prise en charge de l'ancienne colonie française imposent au pouvoir impérial l'acceptation d'une série de compromis qui marqueront pour longtemps le paysage politique et légal de la province de Québec. La coexistence malaisée de deux systèmes juridiques (un droit privé français, un droit public et criminel anglais) a été souvent relevée ${ }^{23}$, et nous n'insisterons pas sur ce point. Il est important cependant de noter que

19. Au sens que lui donne J. Habermas, I'Espace public, Paris, Payot, 1978.

20. Il y a déjà 40 ans, K. Polanyi, avec une puissance d'intuition remarquable, faisait ce constat. K. Polanyi, The Great Transformation, Boston, Beacon Press (1944), 1957.

21. La question du développement de l'appareil d'État anglais au XIX siècle a donné lieu à un important débat historiographique. Voir D. Roberts, Victorian Origins of the British Welfare State, New Haven, Yale University Press, 1960 ; V. Cromwell, «Interpretation of 19th-Century Administration: An Analysis: Victorian Studies, vol. 9, n³, mars 1966, pp. 245-255; P. Richards, «The State and Early Industrial Capitalism: the Case of the Handloom Weavers», Past and Present, n० 83, mai 1979, pp. 91-115; F. Bedarida, «L'Angleterre victorienne, paradigme du laissez-faire?», Revue historique, vol. 261, janvier-mars 1979, pp. 79-98; D.A Wolfe, «Mercantilism, Liberalism and Keynesianism; Changing Forms of State Intervention in Captalist Economies», Revue canadienne de théorie politique et sociale, vol. 5, n“ 1-2, 1981. pp. 6996: P. Corrigan (édit.), Capitalsm, State Formation and Marxist Theory, London, Quartet Books, 1980.

22. M. Miaille, Une introduction critique alt droit, Paris, Maspero, 1976, pp. 159-197.

23. F.P. Walton, «The Civil Law and the Common Law in Canada», Revue légale, N.S., vol. 5, 1899, pp. 329351; W. Smith, «The Struggle over the Laws of Canada, 1763-1783». Canadian Historical Review, vol. 1, 1920, pp. 166- 
la Coutume de Paris et les ordonnances du roi de France forment un héritage juridique à plusieurs égards étroitement apparenté aux règles de la Common Law et aux lois anglaises. Il s'agit en effet, et avant tout, d'un ensemble normatif qui s'est développé à l'ombre du pouvoir royal, et dont l'efficace propre tient à l'extension du pouvoir de justice dévolu, en régime féodal, au souverain. Le droit anglais, comme le droit français, à l'époque, est profondément marqué par la dissolution rapide des juridictions locales ou communautaires qui caractérisaient l'ordre juridique féodal. Le développement jurisprudentiel, le recours de plus en plus fréquent aux cours royales ont bientôt fait que l'appareil de justice occupe une place centrale dans les activités civiles de l'État monarchique. Il en est de même dans les colonies. Encore en 1821, un regard sur les dépenses du gouvernement bas-canadien nous apprend que l'appareil judiciaire mobilise $31 \%$ des dépenses civiles ${ }^{24}$. Cette échelle de dépenses n'est en fait que le symptôme d'une logique de fonctionnement étatique tout à fait particulière.

L'État monarchique et ses transplants coloniaux sont avant tout des organes de commandement/ arbitrage, prolongements ramifiés d'un centre symbolique de la souveraineté: la personne sacrée du roi, trônant au sommet de la hiérarchie féodale. Ce modèle politique est encore bien vivant au XVIII ${ }^{e}$ siècle. La monopolisation graduelle, depuis le XIII ${ }^{e}$ siècle en Europe, des fonctions de justice et de cornmandement, nuancée dans certains cas, tel l'Angleterre, par le droit de regard des élites sociales, est un développement apparaissant surtout comme un palliatif aux contradictions croissantes du féodalisme ${ }^{25}$. Sous cet angle, ce processus de centralisation est un indice de crise beaucoup plus qu'un antécédent obligé de l'État moderne.

Le Québec de la fin du XVII ${ }^{\mathrm{e}}$ siècle est un bel exemple de ce processus: les normes civiles de la Coutume de Paris, développées par les juristes et complétées par les ordonnances du roi de France, connaissent un dépérissement rapide une fois coupées de leurs sources françaises ${ }^{26}$. C'est aussi le cas des règles britanniques de droit public - notamment en matière de contrôle des dépenses pubiques ${ }^{27}$ - et du droit criminel $^{28}$. La crise générale du système normatif, amplifiée par les difficultés d'application d'un cadre juridique hybride, pose de façon aiguë, au Québec, la question de la réforme radicale des principes d'application du droit en vigueur.

Il faut bien voir que ce qui se présente d'abord sous les formes relativement bénignes d'une crise locale d'administration de la justice ${ }^{29}$ et d'une incertitude du droit deviendra très vite une question de souveraineté: la faculté royale de produire et d'appliquer les normes diverses réglementant les rapports sociaux est de plus en plus confrontée à la notion de légitimité démocratique développée par les révolutions américaine et française. Le contenu des normes et les modalités de leur application sont appelés, du coup, à subir des révisions radicales.

186; T. Rinfret, «Reciprocal Influences of the French and English Laws», Revue du Barreau canadien, vol. 4, n" 2, février 1926, pp. 69-85; P.-B. Mignault, «Les rapports entre le droit civil et la «Common Law» au Canada, spécialement dans la province d» Québec», Revue du droit, vol. 11, 1932-1933, pp. 201-21 I : L. Baudoin, «Conflits nés de la coexistence juridique au Canada". McGill Law Journal, vol. 3, 1956-1957. pp. 51-69; A. Morel, «Un exemple de contact entre deux systèmes juridiques: le droit successoral du Québec», Annales de l'Université, N.S., n" 4-5, 1963-1964, pp. 1-14; P. Ferland, "L'intluence du droit anglais sur la procédure civile de la province de Québec». L. Baudoin ef al., Quelques aspects du droit de la province de Québec, Paris, Cujas, 1963, pp. 237-248; J.E.C. Brierley, "The co-existence of Legal Systems in Quebec: «Free and Common Socage» in Canada's 'pays de droit civl'», Cahiers de droit, vol. 20, 1979, pp. 277-287; E. Kolish, Changements dans le droit privé au Québec et au Bas-Canada entre 1760 et 1840): attitudes et réactions des contemporatins, Mtl, Université de Montréal. 1980 (Thèse $3^{*}$ cycle).

24. Le fonctionnement interne de l'État occupe quant à lui $37 \%$ des dépenses civiles. Journal de la Chambre d'Assembláe du Bas-Canada, 1821-1822, append. C. Voir aussi G. Paquet et J.-P. Wallot, Parronage et pouvoir dans le Bas-Canacia (1794-18/2), Montréal, Presses de l'université du Québec, 1973.

25. E.H. Kantorowics. The King's Two Bodies: A Study in Mediaeval Theologv, Princeton. Princeton University Press, 1957: P. Anderson. I'Éfat absolutiste. Paris. Maspero. 1978. vol. 1. pp. 15-61.

26. En France, la restructuration fondamentale que constitue le Code Napolécon de 1804 permet au contraire une mise à jour du droit. Voir A.J. Arnaud, Essai danalyse structurale du Code civil francais. La règle du jeru dans la paix bourgeoise, Paris, L.G.D.J., 1973.

27. H. Brun, la Formation des institutions parlementaires québécoises, 1791-1838. Québec. Presses de l'université Laval, 1970, pp. 201-252

28. Sur ce dernier point, voir J.-M. Fecteau, La Paureté, le crime. I'Éfat. Essai sur F'économie politique du

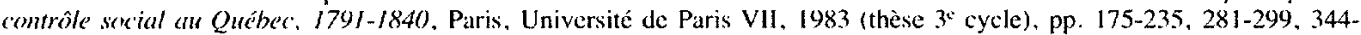
358.

29. Crise latente qui éclate lors de l'enquête de 1787, et encore en 1807. H. Neatby, The Administration of Justice under the Queber Act, Minneapolis, University of Minnesota Press, 1937, pp. 87-174; J.-P. Wallot. «Plaintes contre ladministration de la justice (1807)», Revece d'histoire de l'Amérique fremçerise, vol. 19, mars 1966, pp. 551-560: vol. 20, juin-décembre 1966, pp. 28-43,281-290 et 366-379. 


\section{B. LA MUTATION DU SYSTÈME NORMATIF ET LE POUVOIR PUBLIC (1830-1885)}

C'est cet ébranlement fondamental, qui a pour terme une redéfinition majeure du politique et du légal, qui est à l'cuvre dès les années 1830 au Québec. En situation coloniale, il prendra d'abord la forme d'une révolte générale contre le pouvoir impérial. Les rébellions de 1837-1838 et les tensions politiques qui amènent l'obtention de la responsabilité ministérielle, en 1848, sont à la source même de la mutation que subira le droit dans les 50 ans à venir. Dès 1846 , un auteur anonyme exprime fort bien ce qui est en jeu:

Les conquêtes que les sociétés modernes nous ont faites dans la politique, la science et les arts, l'agriculture, l'industrie et le commerce, ont rendu nécessaire la réformation des vieux codes qui régissaient les sociétés anciennes. Partout l'on a ressenti l'insuffisance des lois faites pour un ordre d'idées et de choses qui n'existent plus, et le besoin de refondre les anciens systèmes et d'en promulguer de nouveaux, afin de se mettre au niveau des progrès de la civilisation ${ }^{30}$.

Le retard relatif avec lequel le Québec s'inscrit dans la logique politique de la démocratie bourgeoise ne rend que plus saisissante et brutale la mutation qui se produit dans l'ordre juridicopolitique. Au cours des trente années qui suivent l'Acte d'Union de 1840, on assiste à une réforme radicale du système politique, de l'appareil administratif, de l'organisation judiciaire et du droit positif. À la source de cette réforme, un phénomène capital: le recentrage du politique dans la formation sociale canadienne. Un recentrage qui prend des formes diverses: contrôle parlementaire et financier de l'exécutif par le biais de la responsabilité ministérielle ${ }^{31}$; décentralisation systématique des pouvoirs de légiférer par la création d'instances municipales ${ }^{32}$; développement des départements administratifs et des pouvoirs de contrôle et d'inspection publics ${ }^{33}$; reconnaissance des responsabilités publiques en matière d'éducation et d'hygiène sociale ${ }^{34}$; développement et systématisation de l'appareil répressif (police et prison); réorganisation et décentralisation du système judiciaire.

Chacune de ces réformes n'apparaît certes pas de façon spontanée: il s'agit de la résultante des multiples tensions politiques qui, depuis l'adoption de l'Acte constitutionnel de 1791, traversent la formation sociale canadienne. Mais l'ampleur et la rapidité des transformations après 1840 méritent que l'on s'attarde à la remarquable concordance des diverses mesures législatives qui les mettent en place. En effet, dans la mouvance de ces réformes radicales, une tendance générale se manifeste; l'autonomisation relative d'une instance publique, d'un champ spécifique d'intervention et de régulation étatique, d'un espace public qui se donne comme l'envers du privé, comme sa condition de possibilité. L'État n'est plus le souverain, est plus que le souverain: il se présente dorénavant comme la cristallisation de la volonté collective, comme la forme d'existence de la collectivité en tant que volonté. C'est cette instance qui légitime et sanctionne désormais les normes structurant les rapports sociaux. Ce cadre défini, les rapports entre individus et groupes divers peuvent maintenant être pensés et définis comme privés. Un tel reclassement des critères de sociabilité et de leur dimension politique n'implique évidemment pas une soumission tranquille de la société civile à l'État, une préséance de la régulation étatique sur la lutte de classes. Le modèle décrit est une nouvelle symbolique du pouvoir, une métaphysique politique des rapports sociaux vus sous le signe du consensus. Mais cette symbolique a une efficace propre, et implique un bouleversement institutionnel qui mettra en place le contexte politique des luttes sociales à venir. C'est pour cette raison qu'une analyse de l'État canadien au XIX ${ }^{2}$ siècle ne peut se satisfaire d'un simple constat de faiblesse institutionnelle. Le modèle de structuration politique mis en place porte en lui-même une logique de développement administratif très claire: la place dévolue à l'État dans ce mode de régulation fait que les modalités de son «intervention», en cas de crise ou de pressions sociales, légitiment l'essor graduel d'un appareil spécifique de réglementation et de gestion.

En matière légale, on assiste alors à la révision radicale et à la fixation de ce que $\mathrm{A}$. $\mathrm{J}$. Arnaud a appelé «les règles du jeu dans la paix bourgeoise». Au Québec, cette révision se déploie dans la plupart des domaines du droit moderne: réforme en profondeur des règles de droit criminel,

\footnotetext{
p. 337 .

30. Anonyme, «De la codification des lo1s du Canada», Revue de législation et de jurisprudence, vol. 1,1846,

31. R.M. Dawson, The Government of Canada, Toronto, University of Toronto Press, 1970, pp. 3-19.

32. J. L'Heureux, «Les premières institutions municipales au Québec, ou 'machines à taxer' ", Cahiers de droit. vol. 20, 1979, pp. 331-356.

33. J.E. Hodgetts, op. cit.

34. Voir notamment P. Carignan, «La place faite à la religion dans les écoles publiques par la loi scolaire de 1841», Revue juridique Thémis, vol. 17, 1982-1983, pp 9-78.
} 
notamment par les lois de Black de $1841^{35}$; abolition du régime seigneurial en $1854^{36}$; codification du droit civil et commercial en $1866^{37}$; codification de la procédure civile en 1867; développement du pouvoir réglementaire des organismes publics en matière municipale, scolaire, sanitaire, carcérale et asilaire; systématisation des procédures d'incorporation des associations diverses et des compagnies ${ }^{38}$. Ce bouleversement du cadre juridique, tout à fait conforme avec l'évolution que connaissent les autres nations occidentales, permet une diffusion systématique du droit dans la formation sociale par un quadrillage plus ou moins serré des rapports sociaux qui donne au droit, comme mode de normalisation, l'autonomie relative qu'il revendique à l'époque contemporaine.

La codification, consolidation ou rénovation du droit auxquelles on assiste à l'époque prennent leur pleine dimension politique dans le pacte confédératif de 1867. Le partage des pouvoirs entre le fédéral et les provinces confirme, en le diffusant entre les divers paliers de gouvernement, cet équilibre délicat qui s'est dorénavant institué entre la légitimité d'une intervention étatique, en terme de droit public, dans le cadre de la démocratie libérale, et le libre déploiement des forces du marché, assuré par le droit privé. C'est cet équilibre qui bientôt manifeste son extrême précarité.

\section{L'ÉQUILIBRE PRÉCAIRE DE LA RÉGULATION CONCURRENTIELLE AU QUÉBEC (1885-1930)}

La fine gradation des responsabilités d'intervention, la subtile distinction entre les tâches dévolues à l'instance publique et le libre déploiement des échanges privés constituent les caractères fondamentaux de ce qu'on a appelé le mode concurrentiel de régulation ${ }^{39}$. Ce modèle des rapports entre Élat et société, inscrit dans le droit et générant une distinction cruciale entre le privé et le public, s'avère le référent fondamental des élites sociales québécoises au tournant du $\mathrm{XX}^{\mathfrak{c}}$ siècle. Il est donc fort tentant d'interpréter la période qui va de la Confédération à la crise de 1929 comme la réalisation de cet idéal sociétal, comme l'âge d'or de la régulation concurrentielle et, conséquemment, du droit privé. La réalité est cependant beaucoup plus nuancée. Ainsi, l'«acte des manufactures» de 1885 a valeur de symbole, et constitue à notre sens une date charnière ${ }^{40}$. Il témoigne en effet de la précarité de l'équilibre juridico-politique rêvé par les tenants de l'économie politique classique. Au-delà des dispositions fort timides que cette loi stipule en matière de protection des travailleurs(ses) en usines, elle dévoile brutalement le caractère utopique de la séparation entre le privé et le public revendiquée par les classes dominantes. La fonction supplétive attribuée à l'intervention de l'État apparaît! en effet, dès ses premières manifestations, comme profondément contradictoire. Trois phénomiènes majeurs sont à la source de cette contradiction:

- D'abord, la source même de la légitimité étatique, à savoir la démocratie de type libéral, est rapidement mise en cause. Les luttes sociales diverses sur les lieux de travail et en milieu urbain, le développement remarquable des organisations ouvrières et des revendications sociales et politiques dont elles sont porteuses, les premières luttes féministes remettent en question les critères discriminants qui confinaient les démocraties libérales du XIX ${ }^{e}$ siècle aux cercles plus ou moins restreints des possédants. L'extension du droit de suffrage, et par là-même de la base sociale obligée de ces démocraties, exerce sur l'instance publique une pression qui deviendra de plus en plus irrésistible ${ }^{4}$. Très vile en effet, les organisations populaires sauront donner une formulation politique aux

35. 4-5 Vict. (1841), c. 24 à 27 . Voir C. Desaulniers, «La peine de mort dans la législation criminelle de 1760 à 1892», Revue générale de droit, vol. 8, 1977, pp. 141-184; A. Morel, «La réception du droit criminel anglais au Québec (1760-1892)», Revue juridique Thémis, vol. 13, 1978, pp. 449-551.

36. J.-P. Wallot, «Le régime seigneurial et son abolition au Canada». Annales historiques de la Révolution française. vol. 41,1969 , pp. 343-371. pp. $521-589$

37. J.E.C. Brierley, «Quebec Civil Law Codification Viewed and Reviewed», McGill Law Journal, vol. 14, 1968.

38. J. Smith et Y. Renaud. Droit québécois des corporattons commerciales, Montréal, Judico, 1974, pp. 5-29; F.E. Labrie et E.E. Palmer, «The Pre-Confederation History of Corporations in Canada», J.S. Ziegel (édit.), Études sur le droit canadien des compagnies, Toronto, Butterworths, 1972, pp. 33-60.

39. A. Lipietz, Crise et inflation, pourquoi?, Paris, Maspero, pp. 169-212; R. Boyer, «La crise actuelle: une mise au point en perspective historique», Critiques de l'économie politique. N.S., $\mathrm{n}^{\text {on }} 7-8$, avril-septembre 1979, pp. 5-36; M. Aglietta et A. Brender, les Métamorphoses de la société salariale, Paris, Calmann-Lévy, 1984.

40. 48 Vict. (1885), c. 32 . Voir R. Chartier. "Contribution à l'histoire de la législation du travail», Relations industrielles, vol. 17, $\mathrm{n}^{\prime \prime} 1,1962$, pp. 43-50.

41. Au Québec. le suffrage universel provincial n'est acquis qu'en 1940. Cependant, le cens électoral est abaissé de façon importante en 1912 et donne accès au vote à la plupart des travailleurs mâles. L'élargissement du droit de vote au fédéral est obtenu dès 1885. Pour une étude comparative de l'avènement du suffrage universel, voir G. Therborn, «The Rule of Capital and the Rise of Democracy», New Left Review. n*103, 1977, pp. 3-41. 
problèmes sociaux qui accompagnent le développement du capitalisme ${ }^{42}$. Elles interrogent donc directement la place de l'État dans la société capitaliste.

- À cette pression politique se greffe un développement considérable de l'appareil administratif étatique ${ }^{43}$. Au tournant du $\mathrm{XX}^{\mathrm{c}}$ siècle se produit un très net accroissement des dépenses publiques à tous les paliers gouvernementaux ${ }^{44}$. De même, on assiste à un essor notable, toutes proportions gardées, des fonctions publiques fédérales et provinciales entre 1867 et $1930^{45}$. L'État, comme structure et appareil de gestion, acquiert donc une visibilité remarquable, et inédite dans la formation sociale québécoise. Cette présence administrative, qui apparaît bien faible à l'observateur actuel, est à l'époque l'indice certain (et menaçant) des contradictions de la société civile libérale.

- Ces contradictions sont rendues manifestes lorsqu'on analyse l'activité législative et réglementaire de l'époque. L'«intervention» de l'État dans les relations de travail et en matière de santé publique, l'adoption des premières lois sociales touchant les accidents de travail, le salaire minimum, la régulation du marché du travail (bureaux de placement), l'assistance publique et les pensions de vieillesse ${ }^{46}$, le financement public de l'infrastructure économique constituent un ensemble de mesures fondamentales qui consacrent la dégradation rapide du modèle de régulation concurrentielle.

C'est dans ce contexte que la très forte résistance d'une partie des classes dominantes québécoises à l'extension du rôle de l'État doit être analysée. Elle manifeste moins un rejet de l'État qu'un rapport à la politique devenu conflictuel et contradictoire ${ }^{47}$. On insiste trop peu cependant sur le fait que ce malaise des élites face à l'État a son exact parallèle dans le domaine du droit. La période qui va de 1885 à 1929 apparaît en effet, à première vue, comme toute dominée par les civilistes ${ }^{48}$. C'est l'époque où les plus éminents juristes de la province développent un véritable culte de «notre Code civil» ${ }^{49}$. Cette vénération affectée a cependant, si l'on y regarde bien, un caractère nettement défensif: elle témoigne d'une résistance acharnée à toute forme d'ingérence dans le corpus sacré de la réglementation civile.

L'ennemi le plus sournois est certes, et depuis longtemps, la Common Law et ses règles d'interprétation qui tendent à envahir la doctrine civiliste ${ }^{50}$. Mais la principale menace réside dans l'activité législative de l'État, qui vient parfois s'attaquer au texte même du Code québécois ${ }^{51}$. Toute modification, par voie statutaire, de la logique constitutive des règles de droit civil est ainsi combattue avec acharnement ${ }^{52}$.

42. C'est le cas des syndicats et partis ouvriers au Québec. Voir l'Action politique des ouvriers québécois (fin du $x I X^{e}$ siècle à 1919), Montréal, Presses de l'université du Québec, 1976.

43. J. E. Hodgetts, The Canadian Public Service. A Physiology of Government, 1867-1970, Toronto, University of Toronto Press, 1973, J.I. Gow, op. cit., pp. 385-391. pp. $13-38$

44. R.M. Bird, The Growth of Government Spending in Canada, Toronto, Canadian Tax Foundation, 1970,

45. Les statistiques concernant les employés gouvernementaux sont peu fiables avant 1900. De 1896 à 1933, on sait que les effectifs de l'administration provinciale québécoise passent de 700 à 8072 employés. Voir J.I. Gow, op. cit., pp. $397-8 ; J$. Desroches, «The Evolution of the Organization of Federal Government in Canada», Administration publique du Canada, vol. 5, 1962, pp. 408-427; V.S. Wilson, Canadian Public Policy and Administration, Toronto, McGraw-Hill, 1981, pp. 324-381

46. M. Pelletier et Y. Vaillancourt, les Politiques sociales et les travailleurs, vol. 1, Montréal, s. édit., 1974.

47. Voir la remarquable mise au point entreprise par R. Heintzman, «The Political Culture of Quebec, 1840-1960», Revue canadienne de science politique. vol. 16, $\mathrm{n}^{\prime} 1$, mars 1983, pp. 3-59.

48. Il faut dire cependant que l'évolution jurisprudentielle globale qui suit l'adoption du Code civil québécois jusqu'en 1930 est un terrain d'étude quasi inexploré. Voir toutefois J. Désy, «Origine et évolution des lois françaises au Canada en matière civile (1869-1919)", dans les Transformations du droit dans les principaux pays depuis 50 ans, Paris, L.G.D.G., 1922, pp. 177-188; P.-A. Crépeault et J.E.C. Brierley, Code civil. Édition historique et critique, Montréal, S.O.Q.U.I.J., 1980

49. Notamment R. Taschereau, «La codification de nos lois civiles», le Droit civil français. Livre-souvenir des journées du droit civil français, Montréal, Barreau de Montréal, 1936, pp. 657-671; J. Désy, op. cit.; P.B. Mignault, «Le Code civil de Québec et son interprétation», Revue du droit, vol. 14, 1935-1936, pp. 583-608; T. Rinfret, op. cit.; P.E. Lamarche, «Le droit civil français sous la domination anglaise» (1911), CEuvres. Hommages, Montréal, Bibliothèque de l'Action française, s.d., pp. 18-49.

50. La création de la Cour suprême en 1875 , avec juridiction d'appel sur les tribuneaux civils de la province de Québec n'a fait qu'accentuer cette tendance. Il en est de même pour les appels au Conseil privé anglais. Voir W.R. Lederman (edit.), The Courts and the Canadian Constitution. Toronto, McClelland \& Stewart, 1964; L. Baudouin, «La réception du droit étranger en droit privé québécois», L. Baudoin et al.., op. cit., pp. 3-70.

civil.

51. Ainsi la loi fédérale de 1890 sur les lettres de change abolit d'un seul coup une centaine d'articles du Code

52. On a une belle illustration de cette résistance dans le rapport Donon de 1930 sur les droits civils des femmes. Voir J. Stoddart, "Quand les gens de robe se penchent sur les droits des femmes: le cas de la Commission Dorion, 1929. 1931 \%, M. Lavigne et Y. Pinard (dir ), Travailleuses et féministes. Les femmes dans la société québécoise. Montréal, Boréal Express, 1983, pp. 307-336. 
Ces menaces à l'«intégrité» du Code civil traduisent l'impact sur le droit des multiples transformations politiques analysées plus haut. L'expansion de la base démocratique, de l'appareil bureaucratique et du champ d'intervention de l'État libéral se manifeste, en matière juridique, par le développement du droit public. Ce phénomène implique, à terme, une crise profonde de la catégorisation normative fixée au milieu du $\mathrm{XIX}^{\mathrm{e}}$ siècle. Si, dans un premier temps, l'ensemble des dispositions statutaires énoncées à la fin du $\mathrm{XIX}^{\mathrm{e}}$ et au début du $\mathrm{XX}^{\mathrm{e}}$ siècle ne semble pas «limiter» la libre application de la réglementation civile, l'extension de ces dispositions nouvelles à des segments de plus en plus vastes de la vie sociale vient, dans un second temps, remettre en cause ce droit privé comme catégorie juridique autonome.

C'est dans le domaine de la «protection sociale» que ce phénomène est le plus manifeste. La réglementation publique des conditions de travail, l'inspection des établissements de charité subventionnés, l'adoption d'un régime de compensation systématique en cas d'accident de travail, le financement d'une partie des frais d'assistance, l'établissement d'un salaire minimum pour les femmes sont un ensemble de mesures qui viennent compléter, nuancer, limiter ou rendre inopérantes les règles établies en droit civil en matière, notamment, de louage d'ouvrage, de responsabilité civile ou de propriété privée.

Ainsi, derrière la défense des principes inscrits dans le Code civil, on peut déceler la résistance désespérée d'une partie des élites dominantes devant les enjeux politiques et sociaux qui soustendent toute remise en question de l'ordre juridique établi. Le régime politique et le système normatif font ainsi face à une crise potentielle de légitimation ${ }^{53}$ qui se manifestera clairement sous le coup de la grande crise de 1929. Les cinquante ans qui précèdent cette crise apparaissent comme les années de gestation de cette contradiction fondamentale qui traverse les rapports entre le droit moderne et l'État contemporain ${ }^{54}$.

\section{CONCLUSION}

$\mathrm{Ce}$ bref essai a eu pour seul but d'expliquer la nécessité d'une relecture de l'histoire du droit en fonction de sa dimension politique. Il ne s'agit évidemment pas de déprécier la qualité des travaux accomplis ou en cours dans ce champ de recherche ${ }^{55}$, mais plutôt d'insister sur le décloisonnement nécessaire de cette discipline. Les analyses de W. Hurst, M. Horwitz, D. Sugarman et, au Canada, de R.C.B. Risk ${ }^{56}$ ont montré la richesse heuristique d'une étude historique du droit confronlée aux réalités économiques. Les problèmes épistémologiques d'une telle approche sont bien sûr importants, et le risque existe de sombrer dans un économisme simplificateur donnant au droit un caractère instrumental excessif ${ }^{57}$.

Une telle démarche de recherche interdisciplinaire reste pourtant nécessaire, notamment dans le domaine des rapports entre État et droit. On ne peut plus se contenter d'une analyse réductrice qui pose l'État comme simple donnée implicite de la production du droit. L'argumentation élaborée dans cet essai postule au contraire un rapport organique entre la place de l'État (et du politique) dans une formation sociale, et le cadre normatif de reproduction de cette formation. Une étude systématique de ce problème devra tenir compte du remarquable développement de la recherche théorique et empirique sur l'État, notamment depuis une dizaine d'années ${ }^{58}$. Cette entreprise de

53. La montée du «réalisme juridique», très nette aux Etats-Unis mais mal documentée au Canada, est la forme que prend, dans le domaine du droit, cette crise de légitimation. Voir K.E. Klare, «Judicial Deradicalization of the Wagner Act and the Origins of Modern Legal Consciousness, 1937-1941». Minnesota Law Review, vol. 62, 1978, pp. 265-339. On ne peut s'empêcher d'être frappé des similarités qui existent entre le réalisme juridique que décrit Klare et I' «instrumentalisme» dans la jurisprudence américaine de la première moitié du XIX ${ }^{\circ}$ siècle, analysé par W.E. Nelson, Americanization of the Common isaw. The Impact of Legal Change on Massachusetts Society, 1760-1830, Cambridge, Harvard University Press, 1975, et surtout par M.J. Horwitz, op. cit. Ce phénomène mériterait d'être étudié plus à fond au Québec.

54. Pour une ébauche de réflexion sur les rapports entre État et droit dans la formation sociale canadienne depuis 1929. voir J.-M. Fecteau et G. Rocher, la Mutation de l'État depuis la crise et la place du droit, Montréal, Centre de recherche en droit public. 1984 (Texte polycopié produit dans le cadre de la Commission Macdonald).

55. On trouvera un bilan, centré sur le Canada anglais, dans D.H. Flaherty, «Writing Canadian Legal History: An Introduction", D.H. Flaherty (édit.), op. cit., pp. 3-42. Voir aussi A. Morel, "Canadian Legal History. Retrospect and Prospect», Osgoode Hall Law'Journal, vol. 21, n'2, juin 1983, pp. 159-164.

56. R.C.B. Risk, "The Law and the Economy in Mid-19th-Century Ontario: A Perspective», D.H. Flaherty (édit.), op. cit., pp. 88-131.

57. On consultera avec profit, sur ce point, la mise en garde de G.R. Rubin et D. Sugarman, «Towards a New History of Law and Material Society in England, 1750-1914», G.R. Rubin et D. Sugarman (édit.), op. cit., pp. 1-123.

58. Pour une bibliographie sommaire, voir J.-M. Fecteau et G. Rocher, op. cit. 
recherche juridico-politique est la condition de possibilité d'une réflexion renouvelée sur les conditions historiques de développement de la logique juridique et de son assise politique.

\section{RÉSUMÉ}

La prépondérance actuelle de la régulation sociale sous une forme juridique présuppose l'existence de cet appareil centralisé qu'est l'État. Le modèle libéral ne sépare pas réellement le public et le privé: libéralisation du marché et réglementation étatique y yont de pair. Dans cette optique, l'auteur examine le développement historique concomitant du droit et de l'État modernes au Québec. Déclin du modèle colonial d'abord (17601830), quand la crise du système normatif devient très vite une question de souveraineté: la faculté royale de produire et d'appliquer le droit se heurte à la notion révolutionnaire de la légitimité démocratique. Ensuite (1830-1885), autonomisation relative du droit comme mode de normalisation et légitimité démocratique de l'État et de ses appareils de réglementation et de gestion. Enfin (1885-1930), remise en question par le mouvement ouvrier de l'idéologie de la séparation du public et du privé et développement rapide du droit public, au point de remettre bientôt en cause le droit privé comme catégorie juridique autonome.

\section{SUMMARY}

The preponderance that social control through legal means now enjoys presupposes the existence of the centralized apparatus of the state. The liberal model does not really separate the public from the private: liberalization of the market and state regulation go hand in hand. The author examines from this point of view the historical development concomitant with modern law and state in Quebec. First, we see the decline of the colonial model (1760-1830), at which time the crisis of the normative system quickly became a question of sovereignty, the power of the king to produce and enforce the law came into opposition with the revolutionary notion of democratic legitimacy. Then (1830-1885), came the period of relative autonomy of the law as a means of normalization, and the democratic legitimacy of the state and of its apparatus for control and management. Finally (1885-1930), came the challenging by the labor movement of the ideology of the separation of public and private. and the rapid development of public law, which soon had the effect that private law was challenged as an autonomous legal category.

\section{RESUMEN}

La preponderancia actual de la regulación social bajo una forma jurídica, presupone la existencia de este aparato centralizado que es el Estado. El modelo liberal no separa realmente lo público de lo privado: liberalización del mercado y reglamentación estatal van a la par. En esta óptica, el autor examina el desarrollo histórico concomitante del derecho y del Estado moderno en Quebec. Ocaso del modelo colonial primeramente (1760-1830), cuando la crisis del sistema normativo se transforma rápidamente en una cuestión de soberanía: la facultad real de producir y de aplicar el derecho se estrella contra la noción revolucionaria de la legitimidad democrática. A continuación (1830-1885), autonomía relativa del derecho como modo de normalización y legitimidad democrática del Estado y de sus aparatos de reglamentación y de gestión. Al fin (1885-1930), cuestionamiento por el movimiento obrero de la ideología que separa lo público de lo privado y desarrollo rápido del derecho público, al punto de poner en causa el derecho privado como categoría jurídica autónoma. 